\title{
Social effects on spatial choice in the radial arm maze
}

\author{
Michael F. Brown, Toni-Moi N. Prince, and Karen E. Doyle \\ Villanova University, Villanova, Pennsylvania
}

\begin{abstract}
Social memory was investigated in the context of a spatial working memory task. Pairs of rats were tested in an eight-arm radial maze. Under most conditions, there was a tendency to choose maze locations that had been visited earlier by the other rat. The possibility that this tendency is produced by common preferences for particular maze locations was ruled out. An opposite tendency to avoid visits to locations that had been visited earlier during the trial by another rat was found only when the maze location contained two pellets (rather than an undepletable supply), the rats' ability to see each other in the maze was restricted to the central arena, and the maze location had been previously visited by the focal rat. The amount of food available in maze locations did not otherwise modulate social influences on spatial choice. The results indicate that memory for a rat's own previous choices is combined with memory for the choices made by another rat.
\end{abstract}

The existence, characteristics, and behavior of conspecifics are important features of most animals' worlds. Although many animals live in relative isolation, many others interact with members of their own species in critically important ways. Given the importance of social interaction, one would expect that the behavior of others serves as the content of many memories and that such social memories would be an important part of our investigations of animal memory. Although there is increasing evidence that learning from other individuals is important for many animals (e.g., Galef \& Laland, 2005; Zentall $\&$ Galef, 1988), very little is known about the existence, properties, or mechanisms of memory for the behavior of other animals.

It is well known that the efficiency of choices made by rats in the radial-arm maze task is increased by memories of the spatial locations that they have already chosen and depleted of food (e.g., Olton, 1978; Olton \& Samuelson, 1976). Recently, work in our laboratory has shown that rats foraging together with their cagemate in a radial-arm maze are also affected by the choices of the other rat and that at least some of these effects are produced by memory for the choices made earlier during the trial by the other rat (Brown, Farley, \& Lorek, 2007; Brown et al., 2008). The nature of the effect produced by choices of the other rat is sometimes an increase in the tendency to visit the locations chosen by it and sometimes an opposite tendency to avoid visits to those locations.

Brown et al. (2007) used a free-choice procedure in which two rats chose simultaneously from the eight locations in the maze, as well as an observer/model procedure in which a subject rat observed a model rat make choices in the maze and later made choices itself. In the latter procedure, the subject rat avoided locations that had been chosen by the model rat. In the free-choice procedure, the results were more complicated. There was a tendency to selectively choose the location that had been most recently visited by the other rat but an opposite tendency to avoid locations visited earlier during the trial by the other rat. Brown et al. (2007) suggested that the physical presence of the other rat on or near the maze location that it had most recently visited functioned as an attractant stimulus. However, rats avoided locations that they remembered to have been visited earlier during the trial by the other rat.

More recently, Brown et al. (2008) reported a series of experiments using the same free-choice procedure used in some of the experiments reported by Brown et al. (2007). Rather than the usual technique of baiting each maze arm with a small amount of food that is depleted when a rat visits the arm, each maze arm was baited with a large number of pellets, so that food remained throughout each trial. Furthermore, the hedonic value of the food was manipulated by baiting some locations with less-preferred grain pellets and other locations with more-preferred sucrose pellets. With one important exception, there was a systematic tendency for the rats in these experiments to visit locations that had been visited by the other rat earlier in the trial. The exception was the case of locations containing the less-preferred grain pellets and visited earlier during a trial both by the focal rat itself and by the other rat. That is, if a rat has information about the relatively low hedonic value of food in a location (by means of its own earlier visit), this rat's memory of visits to that location by a foraging partner decreases its tendency to visit that location. Otherwise, its memory of visits to that location by a foraging partner increases its tendency to visit that

M. F. Brown, michael.brown@villanova.edu 
location. Brown et al. (2008) suggested that this pattern of results requires interaction between information about the rat's own experiences and information about the behavior (choices) of its foraging partner.

Two sets of issues raised by these results motivate the present experiments. The first concerns an alternative to social working memory as an explanation for an increased tendency to visit locations visited earlier by the other rat. The tendency of one rat to choose locations that were chosen earlier by the other rat might not be an effect of the latter on the former but, rather, an artifact of the two rats having similar preferences for the locations (maze arms). It is important to emphasize that the opposite tendency to avoid locations visited by the other rat (found in the model/observer procedures used by Brown et al., 2007, and in the exceptional condition described above by Brown et al., 2008) cannot be explained by such commonality of location preferences. However, commonality of location preferences can explain the tendency to visit locations visited by the other rat. Brown et al. (2008) examined this possibility by looking for commonality of the preferences for the eight maze locations expressed by the two members of each rat pair, in terms of the order in which each location was first visited. The results were mixed. There was evidence that the rats in some pairs tended to visit the eight locations in a similar order. However, that effect did not appear to be sufficient or reliable enough (across experiments) to explain the tendency to visit maze locations that had been visited by the other rat. Instead, Brown et al. (2008) concluded, the rats must be remembering specific locations visited by the other rat earlier in the trial. Their own choices were then modulated by those social working memories. In the first experiment presented below, we provide a direct test of the possibility that common arm preferences can account for the tendency to choose locations chosen by another rat.

The second issue addressed by the present experiments is the discrepancy between the effect of remembered choices made by the foraging partner in the experiments reported by Brown et al. (2007) and those reported by Brown et al. (2008). In both sets of experiments, the location chosen most recently by the other rat was more likely to be visited by the focal rat (consistent with the idea mentioned above that the physical presence of the other rat functions as an attractant). However, in the former experiments, locations visited earlier during the trials by the other rat (and therefore requiring memory to affect behavior) were systematically avoided, whereas, in the latter experiments, such locations were systematically more likely to be visited (with the important exception mentioned above). In Experiments 2, 3, and 4, we examine one possible explanation for the discrepancy. Brown et al. (2008) baited the maze locations with large numbers of pellets, so that food would not be depleted when rats visited the location. Brown et al. (2008) used this unusual procedure to manipulate the hedonic value of maze locations independently of whether the locations had been visited earlier in the trial. The procedure of baiting maze arms such that they are not depleted of food raises the possibility that social memory for visits to locations by the other rat may have increased the tendency to visit those locations (rather than decrease the tendency to visit them), in part because food remained on the maze arms after a visit. The present experiments include manipulation of this contingency to determine whether it modulates the effect of memories for choices made by the other rat.

\section{EXPERIMENT 1}

Reports from our laboratory have explained the tendency of a rat to choose maze arms in the radial maze that were visited earlier by a foraging partner in terms of an influence of the foraging partner on choices made by the focal rat (Brown et al., 2007; Brown et al., 2008). However, an alternative possibility is that the tendency to visit the most recent location chosen by the other rat is an artifact of common preferences for particular maze arms. To the extent that rats prefer locations (maze arms) in the apparatus and those preferences are consistent across rats (or, at least, across cagemates), a tendency to visit maze arms that were visited earlier by the other rat would be expected, even if the rats do not have any influence on each other's choices.

Brown et al. (2008) examined this possibility by looking for similarities in the orders in which two rats making choices together chose particular maze arms. The results were equivocal. In one experiment, there was no evidence of commonality of the maze-arm preferences, but, in a second experiment, there were some pairs of rats that tended to choose maze arms in a similar order. Even the commonality that was found, however, is theoretically ambiguous. Rats could visit maze arms in a similar order either because of common preferences for maze locations or because of social influences of one rat's (or both rats') choices on the other rat.

Experiment 1 was designed to determine whether pairs of rats have common location preferences that are independent of social effects and could therefore provide an alternative to social influences as an explanation of the tendency to visit locations that were visited by another rat. To this end, naive pairs of rats were tested in the same maze used in the experiments of Brown et al. (2008). On half of the trials, they were tested separately, and on the remaining half, they were tested together. The extent to which members of each pair had common preferences for the eight maze locations was measured and compared under these two conditions. If there are common location preferences independent of social influences, they should be present even when the rats are tested separately. On the other hand, to the extent that common arm preferences are produced by social influences, location preferences should be similar when the rats are tested together to a greater extent than when they are tested separately.

\section{Method}

Subjects. The subjects were 12 male Sprague Dawley rats, obtained from Harlan-Sprague-Dawley, Inc. (Indianapolis, IN) as weanlings. They had been housed in pairs and were provided with free access to water and food until they were 12 weeks old. Experimental trials occurred during the dark phase of a 12:12-h light:dark cycle. One rat in each pair was marked on its tail and back to facili- 
tate identification. The rats were placed on a restricted diet, and their weights were monitored throughout the experiment, such that they were maintained at $85 \%-95 \%$ of free-feeding weight. Beginning 3 days before being first placed in the maze, the rats were given several sucrose pellets in their home cages daily.

Apparatus. The apparatus was a radial maze with eight arms. The central arena was constructed of plastic, $25 \mathrm{~cm}$ tall, $46 \mathrm{~cm}$ in diameter, and had a floor consisting of a thick layer of the same wood chips used in the rats' home cages. Eight equally spaced circular holes $(10.0 \mathrm{~cm}$ in diameter and centered $6.5 \mathrm{~cm}$ above the floor) led to the arms, which were tubes constructed of thin, transparent plastic (PETG), $10 \mathrm{~cm}$ in diameter and $90 \mathrm{~cm}$ long.

Food cups were mounted on pedestals just beyond the ends of the maze arms. These food cups were constructed of translucent plastic and were $10 \mathrm{~cm}$ in diameter and $6.5 \mathrm{~cm}$ deep. Each food cup lid had a $3.5-\mathrm{cm}$-diameter hole cut in its center to allow access to the food pellets with which the cups were filled (45-mg sucrose pellets, P. J. Noyes, Inc., Product PJFSC000045). The height of the pedestals was such that the food cup lids were on the same horizontal plane as the bottom of the maze arms

The maze was centered in a $36.1 \times 35.3 \mathrm{~m}$ room with a variety of objects around the perimeter and was illuminated by fluorescent tubes. A video camera was mounted above the central arena of the maze and allowed the experimenter to monitor the behavior of the rats in the central arena from his or her station in one corner of the room.

Preliminary training. Three trials of preliminary training preceded the experiment. These preliminary trials were conducted just like the together trials described below, except that sucrose pellets were scattered in the central arena and on the arms of the maze. The rats were allowed to explore the maze and to consume pellets for 10 min.

Experimental testing. The rats were tested in two kinds of trials. In together trials, cagemates were tested at the same time. In separate trials, the rats were tested individually. These trials were otherwise identical to the together trials. One trial per day was conducted (5-6 days per week).

The maze was in one of eight equally spaced orientations (randomly chosen each day) for each trial. Thus, spatial locations and physical maze arms corresponded within but not across trials. On each trial, the rat or pair of rats was first placed in the central arena of the maze and was allowed to choose maze arms until 6 min elapsed. A choice was defined as when all four paws were on the surface of the maze arm. The experimenter recorded the sequence of arms chosen (and which rat made each choice, in the case of together trials). Trials were videotaped using a camera mounted directly above the central arena of the maze, and the videotape record was consulted as needed to resolve any ambiguities in the choice sequence.

Following preliminary training, 12 together trials and 12 separate trials occurred for each rat. For counterbalancing purposes, these were structured as four blocks of 6 trials, with 3 trials of each type (randomly ordered) within each block.

\section{Results}

To measure the extent to which the maze-arm preferences of rats in each pair were similar, we calculated the rank order of the maze arms visited by each rat and then determined the correlations among the mean rank orders of members of the pairs when they were tested together and separately in the maze (Brown et al., 2007). For each rat, the eight maze locations were ranked (from 1 to 8 ) in terms of the sequence of the rat's first visit to each of them. In (rare) cases of trials in which a rat did not visit all eight locations, the location(s) not visited were assigned ranks that equaled the mean of the remaining ranks (e.g., if two maze arms were not visited, then those locations both received a rank of $[7+8] / 2=7.5$ ). The correlation
(Spearman's $r$ ) between the ranks of the two members of each pair was then calculated for each trial (together trials) or pair of trials (separate trials occurring on the same day). These correlations were then compared using a 2 (separate vs. together condition) $\times 4$ (trial block) ANOVA.

Figure 1 shows the mean correlations as a function of condition and trial block. The ranks were correlated to a greater extent in the together trials than in the separate trials $[F(1,5)=28.7, p<.01]$. There was no effect of trial block $[F(3,15)<1]$. The interaction between trial blocks and trial condition did not reach significance $[F(1,5)=3.09, p=$ $.06]$. The mean correlation between the ranks (collapsed over trial blocks) was not significantly different from zero in the case of separate trials $[t(5)=1.2]$ but was significantly positive for together trials $[t(5)=5.3, p<.01]$.

\section{Discussion}

The results of this experiment provide clear evidence that the tendency to visit locations that were visited earlier during a trial by the other rat is a social effect rather than a result of common location preferences. The importance of this finding is that an increased tendency to visit places that were visited earlier during a trial by the other rat, just like a decreased tendency to visit such places, is based on the social influence of one rat's previous choices on the subsequent choices made by the other rat.

It should be pointed out that the lack of common arm preferences found in the present experiment when the rats were tested separately does not mean that common arm preferences never occur. However, in the maze and extramaze environment used in these experiments, there apparently were no systematic preferences for a particular maze arm across rats. This is likely to be true of the earlier experiments reported by Brown et al. (2007; Brown et al., 2008), which involved the same lab room and extramaze environment used in the present experiments.

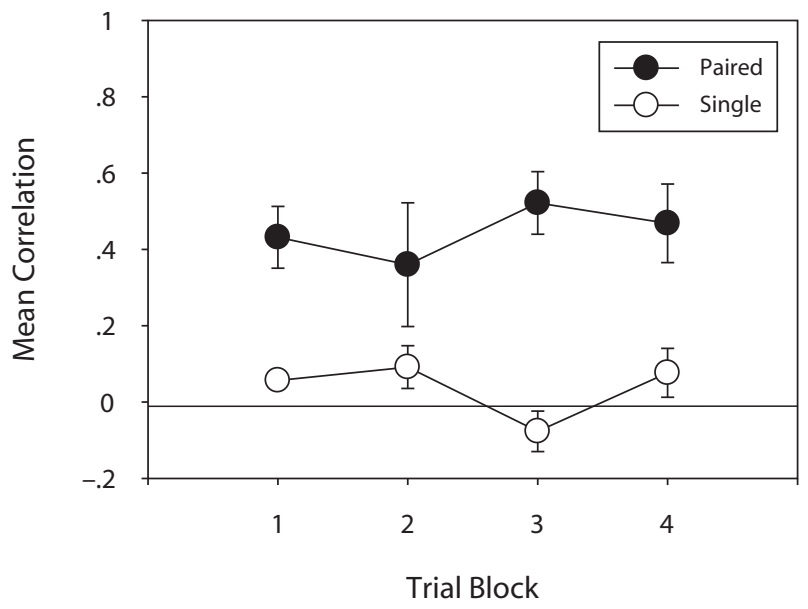

Figure 1. The mean correlation of the orders in which maze locations were visited by the rats in each pair during Experiment 1. The two rats were tested either together or separately and the data are shown over four trial blocks. Error bars show one standard error of the mean. 


\section{EXPERIMENT 2}

The results of Experiment 1 confirm that a tendency to selectively visit locations previously visited by another rat, like a tendency to avoid visits to locations previously visited by another rat, is produced by a social influence of one rat's behavior on the other rat. As described above, both tendencies have been found under circumstances that make memory for the choices made by the other rat the most likely mechanism of the social influence. However, the factors that determine whether previous choices by a foraging partner increase or decrease the tendency to visit a location are not clear. A tendency to avoid locations visited earlier during the trial dominated in the experiments reported by Brown et al. (2007), whereas a tendency to selectively visit locations visited earlier during the trial dominated in the experiments reported by Brown et al. (2008). One potentially critical difference between the procedures used in the two sets of experiments is the amount of food with which the maze arms were baited. The former experiments involved the typical procedure of baiting maze arms with a small amount of food, which was depleted when a rat visited the maze arm. In the latter experiments, the maze arms were baited with a large amount of food, which was not depleted when the rats visited the arm.

The present experiment was similar to those of Brown et al. (2008) in terms of experimental techniques and design. However, instead of manipulating the value of the food reinforcement available in maze locations by using less-preferred (grain pellet) and more-preferred (sucrose pellet) food items, the amount of food available in maze locations was manipulated. During some trials, the maze locations each contained an unlimited supply of sucrose pellets, and during other trials, the locations contained two sucrose pellets. This manipulation of food amount rather than of food type may result in a larger difference between the values of food items in the two kinds of locations than was the case in the Brown et al. (2008) experiments. More critically, manipulation of the amount of food available in locations results in very different contingencies between visits to maze locations and the content of those locations. When baited with an unlimited supply of pellets, the content and value of maze locations is not changed by a visit. However, as is the case in most experiments using the radial maze, a visit to a location baited with two pellets results in the absence of food in that location during subsequent visits. We expected that the former contingency would produce a tendency to visit locations that were previously visited by the other rat (as in Brown et al., 2008) but that the latter contingency would produce the opposite tendency to avoid locations that were previously visited by the other rat.

\section{Method}

Subjects. The subjects were 10 male Sprague Dawley rats, obtained from the same source and maintained in the same manner as were the rats in Experiment 1.

Apparatus. The apparatus was the same radial maze used in Experiment 1, with a modification of the food cups at the end of each maze arm. One of two types of opaque white plastic lids was on these cups, depending on experimental condition. In the manypellets condition, the food cups were covered by the same lids used in Experiment 1 (i.e., there was a 3.5 -cm-diameter hole cut in their center to allow access to the food pellets with which the cups were filled. In the two-pellets condition, intact lids (without holes) were placed on the food cups, and two pellets were placed on the center of each lid.

Preliminary training procedure. For three trials, pairs of rats (cagemates) were placed in the maze for 10 min using the procedure described below, except that pellets were also scattered inside the maze arms and in the central arena to facilitate exploration of the maze. Results presented below do not include behavior during this preliminary training phase.

Testing procedure. The maze was in one of eight equally spaced orientations (randomly chosen each day) for each trial. Thus, spatial locations and physical maze arms corresponded within but not across trials. On half of the trials, each maze arm was baited with two sucrose pellets, placed on the center of the intact lid of the food cup. On the remaining half of the trials, the food cups were filled with sucrose pellets, and the lids with holes allowed access to them. The condition (two pellets or many pellets) in force on each trial was randomly selected, with the constraint that there were 6 trials in each condition in each of two blocks of 12 trials.

On each trial, the two rats were first placed in the central arena of the maze and were allowed to choose maze arms until 6 min had elapsed. A choice was defined as when all four paws were on the surface of the maze arm. The experimenter recorded the sequence of arms chosen.

\section{Results}

The number of choices among the first eight choices of each trial made was determined as a function of five variables: (1) the previous visit status of the focal rat (i.e., whether the location chosen had been chosen earlier in the trial by the focal rat), (2) the previous visit status of the other rat (i.e., the location chosen had not been chosen earlier in the trial by the other rat, was the other rat's most recent choice, or had been chosen by the other rat earlier in its choice sequence), (3) whether the maze arms were baited with two or many sucrose pellets, and (4) trial block. The number of maze locations in each of these same categories at the time these choices were made was also determined. These counts of choices and locations available were then used to determine the obtained and expected proportions, respectively.

We used the analytic technique developed by Brown et al. (2007) and Brown et al. (2008) to measure choice tendencies in terms of these obtained and expected proportions. For each rat, the proportions of choices made to maze locations in each of the 24 categories defined by the variables above was determined: 2 (focal rat status) $\times 3$ (other rat status) $\times 2$ (food amount) $\times 2$ (trial block). To provide estimates of the corresponding proportions that would be expected on the basis of chance, the number of locations (maze arms) in each of these same 24 categories at the time those choices were made was also determined. The counts of choices and locations from each trial were made twice, once from the perspective of each of the two rats. The two corresponding sets of proportions - of choices made (obtained proportions) and of maze locations available to choose (expected proportions) - were then compared in order to assess choice tendencies. Cal- 


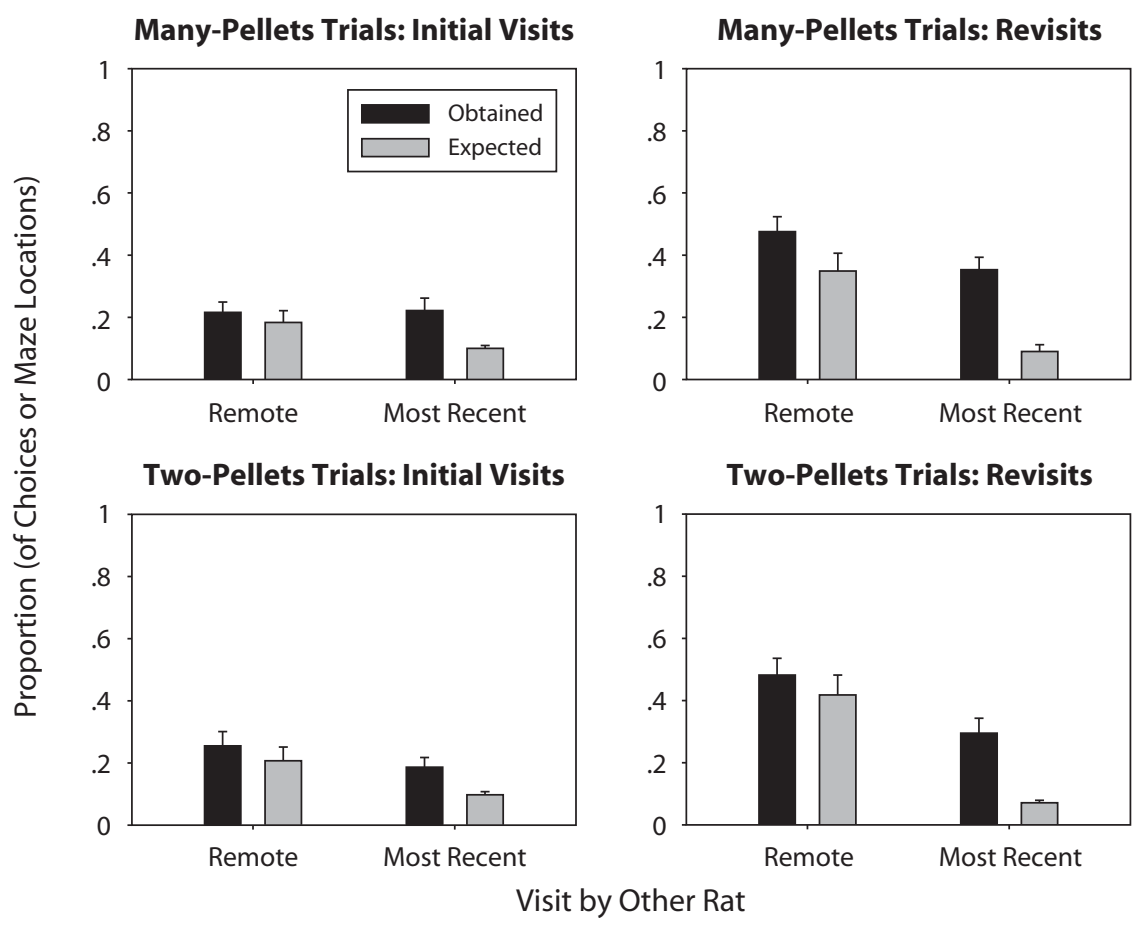

Figure 2. Mean proportions of maze locations chosen (obtained proportions) and proportions of maze locations available at the time those choices were made (expected proportions) during Experiment 2. Each panel shows the proportions for the most recent choice of the other rat and the choice made by the other rat earlier in the trial. The values shown are the means (over rats) of the resulting proportions for initial visits to a maze location by the focal rat (left panels) and revisits to maze locations by the focal rat (right panels) during the trials in which the maze locations were baited with many pellets (top panels) or with two pellets (bottom panels). Error bars show one standard error of the mean.

culation and analysis of these proportions were equivalent to those detailed in Brown et al. (2008; see particularly pp. 328-331 and Appendix 1 for a detailed description and justification of the analytic technique).

Preliminary analyses failed to identify any differences in the pattern of results for the two trial blocks, and so that variable is not included in the analyses described below. Figure 2 shows mean (over rats) proportions of choices made and choices expected (locations available) to the categories that include visits by the other rat earlier in the trial. The critical comparisons in all of the data analyses presented below are differences between these obtained and expected proportions for each category of maze location. The expected and obtained proportions of choices were calculated over the first eight choices for the focal rat's initial choices (i.e., the first visit to a particular maze location) and for revisits of maze locations made during the first eight choices.

An ANOVA compared the proportions of choices obtained with the proportion expected (i.e., the proportion of locations available to be chosen) when the other rat had previously chosen the location as a function of whether the other rat's choice was its most recent choice (or a more remote choice), whether the focal rat had previously chosen the location, and the food type with which the location was baited - that is, a 2 (other status: most recent vs. remote) $\times 2$ (focal status: initial visit vs. re- visit) $\times 2$ (food amount: two pellets vs. many pellets) $\times$ 2 (proportion type: choices vs. maze locations available) ANOVA. Note that only the factors involving comparisons of the obtained proportion of choices and the proportion of maze locations have any theoretical or interpretive meaning, and so only those factors are considered in this article. The overall proportion of choices to locations previously visited by the other rat (.311) was greater than the proportion expected (.189) [proportion type, $F(1,10)=$ 48.7]. There was a proportion type $\times$ focal status interaction $[F(1,10)=16.9]$. No other interactions involving proportion type were significant, although the proportion type $\times$ other status interaction term approached significance $[F(1,10)=3.65, p=.09]$.

The proportion type $\times$ focal status interaction was probed by performing two other status $X$ food amount $X$ proportion type ANOVAs, one for initial visits by the focal rat and the other for revisits by the focal rat. In both cases, the proportion of visits to locations visited by the other rat was larger than the proportion expected $[F(1,9)=48.9$ for initial visits; $F(1,9)=37.3$ for revisits]. In both cases, there were no significant interactions involving proportion type. Thus, the proportion type $\times$ focal status interaction indicates a larger tendency to visit locations visited by the other rat in the case of revisits by the focal rat. However, the tendency to visit locations previously visited by the other rat is present under all conditions. 


\section{Discussion}

There was a tendency to visit maze locations that had been visited by the other rat. That tendency was larger in the case of revisits to maze arms by the focal rat than it was for initial visits to maze arms. There was no evidence that the tendency to visit locations that had been visited by the other rat differed between the most recent choice of the other rat and choices it made earlier in the trial. Most critically, there was no indication that the tendency to visit maze arms visited earlier by the other rat varied as a function of how much food was located on the maze arms. This indicates that the tendency to visit locations that were visited earlier by the other rat does not depend on the absence of a contingency between a visit to a location by the other rat and depletion of food from the location. Even when locations are not depleted of food when visited, that tendency remains.

\section{EXPERIMENT 3}

Experiment 2 provides no evidence that the amount of food available on maze arms (and therefore a contingency between arm visits and food presence) modulates the influence of previous visits by the other rat. However, it remains possible that the contingency between the other rat's arm visits and the presence of food in the locations it visits has a long-term effect that is acquired over trials. If so, the within-subjects manipulation of food amount in Experiment 2 was not adequate to reveal such an effect. Thus, Experiment 3 is an additional attempt to determine whether the amount of food on each maze arm modulates social influences on choice in the radial maze. In Experiment 3, pairs of rats were divided into two experimental groups. For one group, the maze arms were baited with two sucrose pellets prior to each trial. For the other group, the maze arms were baited with a large cache of sucrose pellets, which was not depleted by visits to the arms.

\section{Method \\ Subjects and Apparatus. The subjects were 16 male Sprague Dawley rats, obtained from the same source and maintained in the same manner as the rats in Experiments 1 and 2. The apparatus was the same radial-arm maze used in Experiments 1 and 2. \\ Procedure. The eight cagemate pairs of rats were randomly as- signed to two experimental groups, with the constraint that there were four pairs of rats in each group. For the many-pellets group, the food cups were filled with pellets at the beginning of each trial. The lids with $3.5-\mathrm{cm}$ holes were always present on the food cups when rats in this group were tested, allowing access to pellets on every maze arm throughout each trial. For rats in the two-pellets group, the intact lids were on the food cups, and two pellets were placed on the lid of each food cup prior to each trial. \\ Preliminary training and testing phases were conducted just as were those in Experiment 2. The rats were then tested in 40 trials, conducted just as were those in Experiment 2, with the exception that the amount of food with which maze locations were baited was varied across subjects. The proportions of choices and locations available to choose were examined in terms of the same variables as was done in Experiment 2 in two blocks of 20 trials each.}

\section{Results}

As for Experiment 2, the proportions were calculated on the basis of choices made (and corresponding locations available) during the first eight choices of each trial. The means of the resulting proportions of choices obtained and expected are shown in Figure 3. Unlike in Experiment 2, differences in the pattern of results for the two trial blocks were detected, so, in this case, the data are shown as a function of trial block.

An ANOVA compared the proportions of choices obtained with the proportion expected (i.e., the proportion of locations available to be chosen) when the other rat had previously chosen the location as a function of whether the other rat's choice was its most recent choice (or a more remote choice), whether the focal rat had previously chosen the location, trial block, and the food type with which the location was baited - that is, a 2 (other status: most recent vs. remote) $\times 2$ (focal status: initial visit vs. revisit) $\times 2$ (food amount: two pellets vs. many pellets) $\times$ 2 (trial block) $\times 2$ (proportion type: choices vs. maze locations available) mixed ANOVA, with all factors except food amount being within subjects.

As in Experiment 2, the overall proportion of choices to locations previously visited by the other rat (.302) was greater than the proportion expected on the basis of location available that had been visited by the other rat (.200) [proportion type, $F(1,14)=79.3]$. The significant interactions involving proportion type were proportion type $\times$ food amount $[F(1,14)=12.5]$, proportion type $\times$ food amount $\times$ block $[F(1,14)=4.73]$, proportion type $\times$ food amount $\times$ focal status $[F(1,14)=16.7]$, proportion type $\times$ other status $[F(1,14)=6.0]$, and proportion type $\times$ focal status $\times$ other status $[F(1,14)=6.8]$.

To understand the nature of these interactions, we conducted a proportion type $\times$ other status ANOVA at each level of food amount, focal status, and trial block (i.e., for the data represented in each panel of Figure 3). The results of these ANOVAs are shown in Table 1. The proportion of visits was significantly greater than expected in every case, indicating a consistent tendency to visit maze locations that had been visited by the other rat. There was a significant interaction between proportion type and other status in the case of the many-pellets group for revisits to maze arms during Block 2. The form of that interaction was that the tendency to visit maze locations that had been visited by the other rat was stronger for the location most recently visited by the other rat.

\section{Discussion}

The results of this experiment replicated those of Experiment 2. There was a consistent tendency to visit maze arms that had been visited by the other rat earlier during the trial, regardless of the amount of food with which the maze arms were baited. In the present experiment, the amount of food with which the maze arms were baited was manipulated across subjects, so that individual rats were exposed to consistent contingencies involving food amount. Even so, there was no evidence that the amount of food with which the maze locations were baited had any effect on choices. This finding strengthens the conclusion that the social influence of one rat's choices on choices made by the other rat is not determined or modulated by the contingency between the other rat's visits and the re- 
Block 1

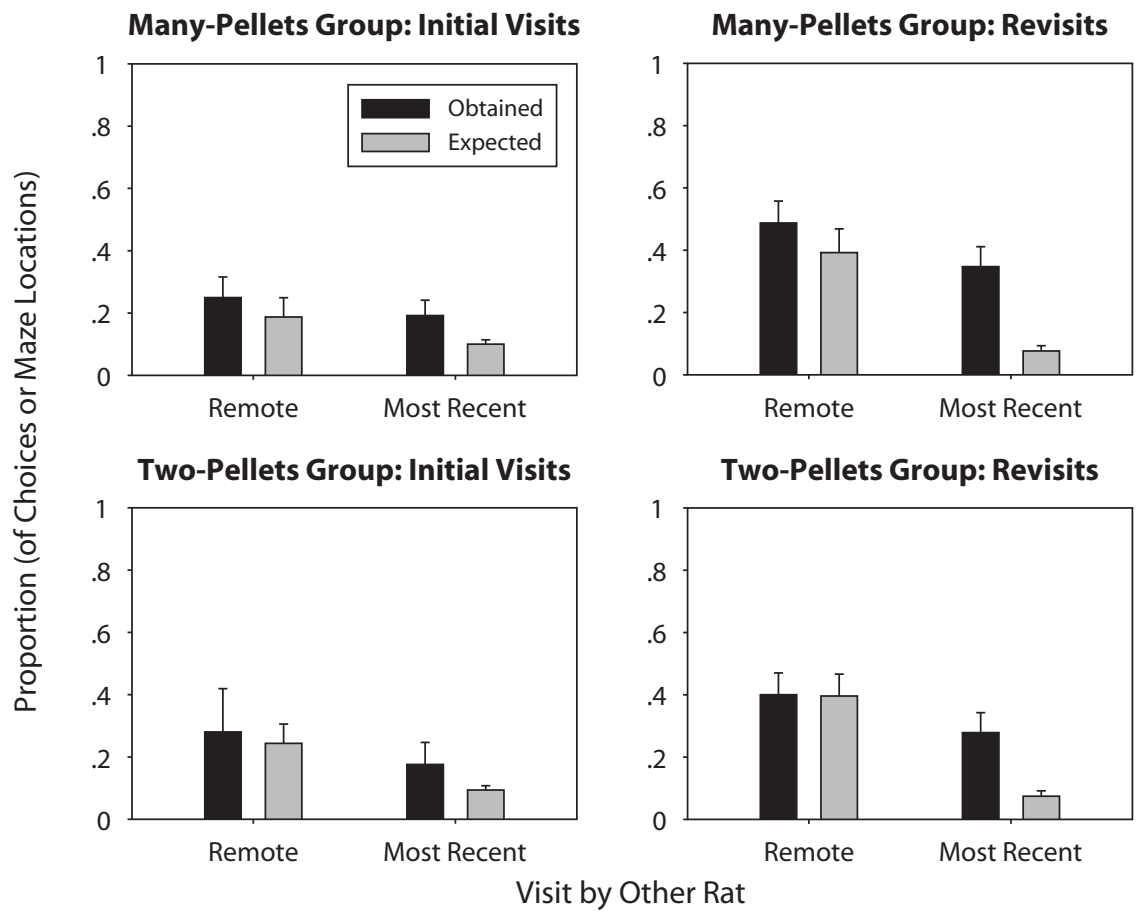

B

Block 2

Many-Pellets Group: Initial Visits

Many-Pellets Group: Revisits
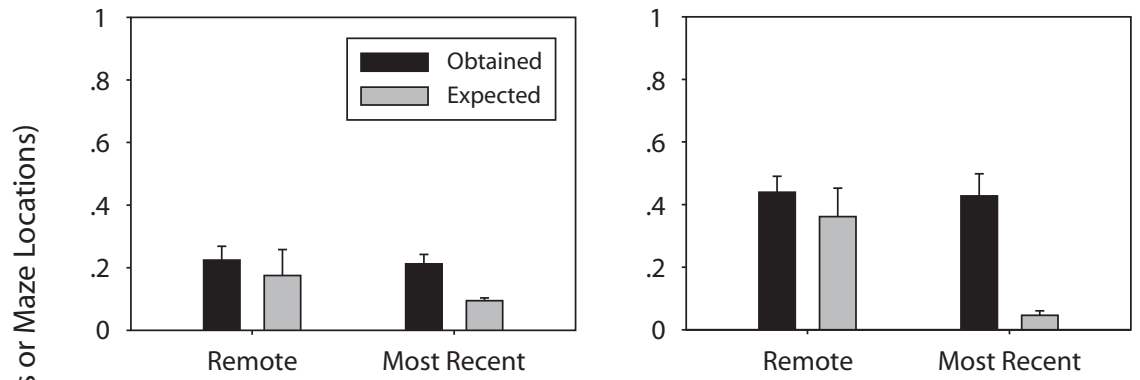

Two-Pellets Group: Initial Visits

Two-Pellets Group: Revisits
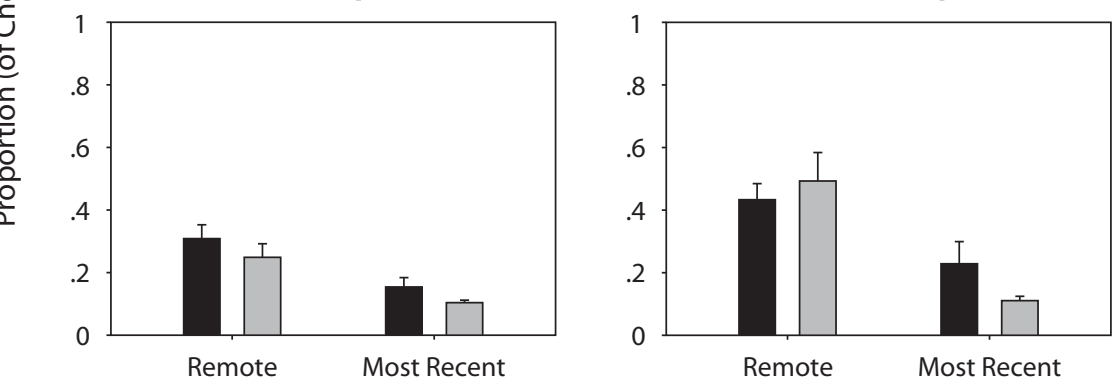

Visit by Other Rat

Figure 3. Mean proportions of maze locations chosen (obtained proportions) and proportions of maze locations available at the time those choices were made (expected proportions) during Block 1 (A) and Block 2 (B) of Experiment 3. Each panel shows the proportions for the most recent choice of the other rat and the choice made by the other rat earlier in the trial. The values shown are the means (over rats) of the resulting proportions for initial visits to a maze location by the focal rat (left panels) and revisits to maze locations by the focal rat (right panels) in the experimental group for which the maze locations were baited with many pellets (upper panels in both A and B) or with two pellets (lower panels in both A and B). Error bars show one standard error of the mean. 
Table 1

\begin{tabular}{|c|c|c|c|c|}
\hline \multicolumn{5}{|c|}{$\begin{array}{c}\text { Table } 1 \\
\text { Results of ANOVAs for Experiment } 3\end{array}$} \\
\hline $\begin{array}{l}\text { Food } \\
\text { Amount } \\
\text { Group }\end{array}$ & $\begin{array}{c}\text { Trial } \\
\text { Block }\end{array}$ & $\begin{array}{l}\text { Focal Rat } \\
\text { Visit Status }\end{array}$ & Proportion & $\begin{array}{c}\text { Other Rat Visit } \\
\text { Status } \times \text { Proportion }\end{array}$ \\
\hline Many pellets & 1 & Initial visits & $F(1,7)=13.0^{*}$ & $F(1,7)<1.0$ \\
\hline Many pellets & 1 & Revisits & $F(1,7)=31.5^{*}$ & $F(1,7)=2.4$ \\
\hline Two pellets & 1 & Initial visits & $F(1,7)=20.0^{*}$ & $F(1,7)=2.5$ \\
\hline Two pellets & 1 & Revisits & $F(1,7)=26.9^{*}$ & $F(1,7)=1.8$ \\
\hline Many pellets & 2 & Initial visits & $F(1,7)=38.6^{*}$ & $F(1,7)=4.6$ \\
\hline Many pellets & 2 & Revisits & $F(1,7)=21.0^{*}$ & $F(1,7)=14.8^{*}$ \\
\hline Two pellets & 2 & Initial visits & $F(1,7)=23.4^{*}$ & $F(1,7)<1.0$ \\
\hline Two pellets & 2 & Revisits & $F(1,7)=3.8^{*}$ & $F(1,7)<1.0$ \\
\hline
\end{tabular}

${ }^{*} p<.05$.

sulting presence/absence of food in the visited location. Regardless of that contingency, rats tend to visit locations visited earlier by the other rat under the conditions of the present experiments.

\section{EXPERIMENT 4}

A potentially important detail of the apparatus used in the previous experiments is that the maze arms were transparent, possibly allowing the two rats to see each other throughout the trial. The same maze with transparent arms was used by Brown et al. (2008, Experiment 2). They also found a tendency to visit locations that had been visited by the other rat in this maze. Furthermore, the magnitude of the tendency to visit locations that had been visited earlier by the other rat appeared to be greater in the experiment involving the maze with transparent arms than in a corresponding experiment (their Experiment 1) involving a similar maze but with opaque arms.

Brown et al. (2008) suggested that the physical presence of the other rat consistently attracts the focal rat to its location, whereas social working memory for choices made by the other rat can result in either attraction to or avoidance of the locations chosen by it. If the transparent arms of the present maze allow the rats to see each other during a larger portion of the trial than do the opaque maze arms used by Brown et al. (2007) and in the first experiment reported by Brown et al. (2008), it is possible that the attraction to the other rat dominated a tendency to avoid places visited earlier by the other rat. This provides a potential explanation for the discrepancies in how choices made by one rat affect the choices made by another rat. Perhaps as long as the other rat is detected as physically present, its present and former locations are more rather than less attractive.

The present experiment was designed to test this possibility, using the design of Experiment 3, but with an additional independent variable. On half of the trials, the transparent maze arms were used, and, thus, the procedure replicated that of Experiment 3. On the remaining trials, the maze arms were covered by opaque sleeves that prevented visual information from being obtained while a rat was on the maze arm. This allowed performance with and without access to visual information while the rats traveled the length of the maze arm (including sight of the other rat) to be directly compared. It is important to note that, even in the sleeve condition, visual cues from the other rat were available when both rats were in the central arena (when the other rat was making its choices). This was intended to be equivalent to the situation in place for the maze with opaque arms that was used by Brown et al. (2007) and in Experiment 1 of Brown et al. (2008).

\section{Method}

Subjects and Apparatus. The subjects were 16 male Sprague Dawley rats, obtained from the same source and maintained in the same manner as were the rats in the earlier experiments. The apparatus was the same radial-arm maze used in the earlier experiments. However, in some trials, the maze arms were rendered opaque and dark by the use of black felt tubes (sleeves) that covered each of the arms from its junction with the central arena to the end of the arm.

Procedure. The eight cagemate pairs of rats were randomly assigned to either the many-pellets group or the two-pellets group, with the constraint that there were four pairs of rats in each group.

Preliminary training and testing phases were conducted as were those in Experiment 3. The rats were then tested in 24 trials, conducted as were those in Experiment 3, with the exception that the experimental design called for the maze-arm sleeves to be present on half of the trials. Because of experimenter error, however, each rat had 11 trials with the sleeves in place and 13 trials without the sleeves. The data were analyzed in two blocks of 12 trials each.

\section{Results}

As for the earlier experiments, the obtained and expected proportions of choices of maze arms that had been previously visited by the other rat were calculated. The means of the resulting values are shown in Figure 4. No significant effects involving trial block were found, and, therefore, the presentation and analyses presented below do not include that factor.

An ANOVA compared the proportions of choices obtained with the proportion expected (i.e., the proportion of locations available to be chosen) when the other rat had previously chosen the location as a function of whether the other rat's choice was its most recent choice (or a more remote choice), whether the focal rat had previously chosen the location, the food type with which the location was baited, and whether the sleeves covering the maze arms were present - that is, a 2 (other status: most recent vs. remote) $\times 2$ (focal status: initial visit vs. revisit) $\times 2$ (food amount: two pellets vs. many pellets) $\times 2$ (sleeves present vs. absent) $\times 2$ (proportion type: choices vs. maze locations available) mixed ANOVA, with all factors except food amount treated as within subjects. 
A

No Sleeves Present

Many-Pellets Group: Initial Visits

Many-Pellets Group: Revisits
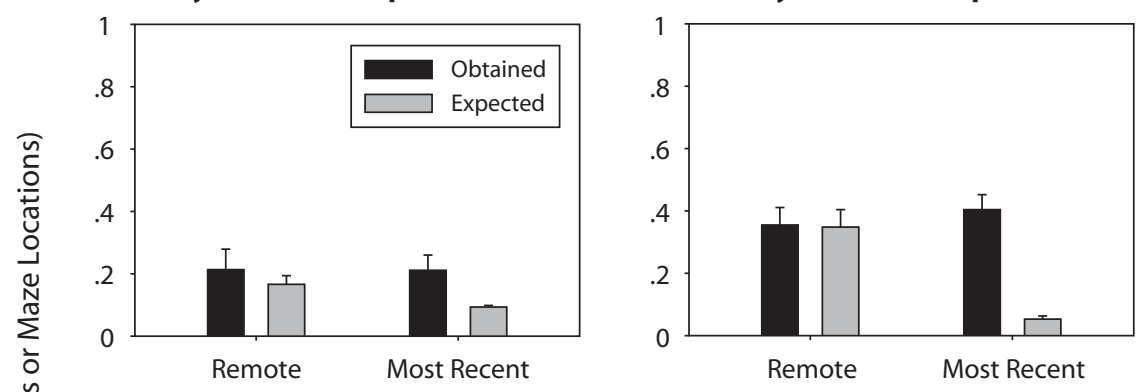

Two-Pellets Group: Initial Visits

Two-Pellets Group: Revisits
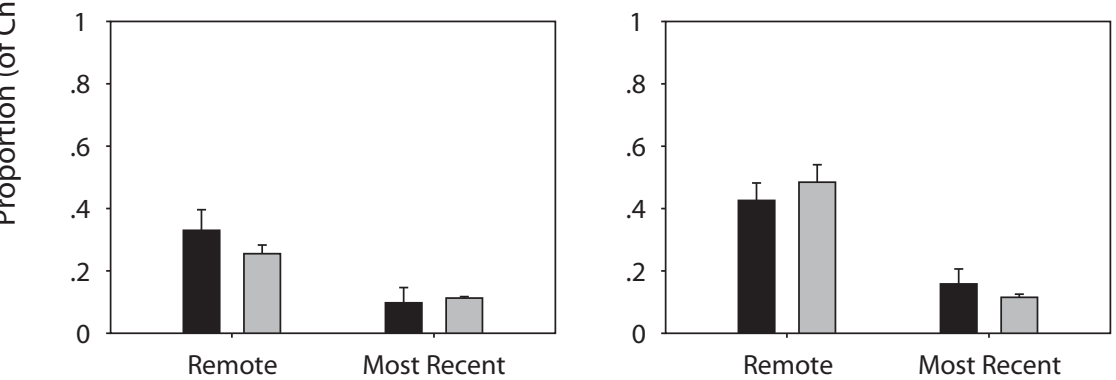

Visit by Other Rat

B

Sleeves Present

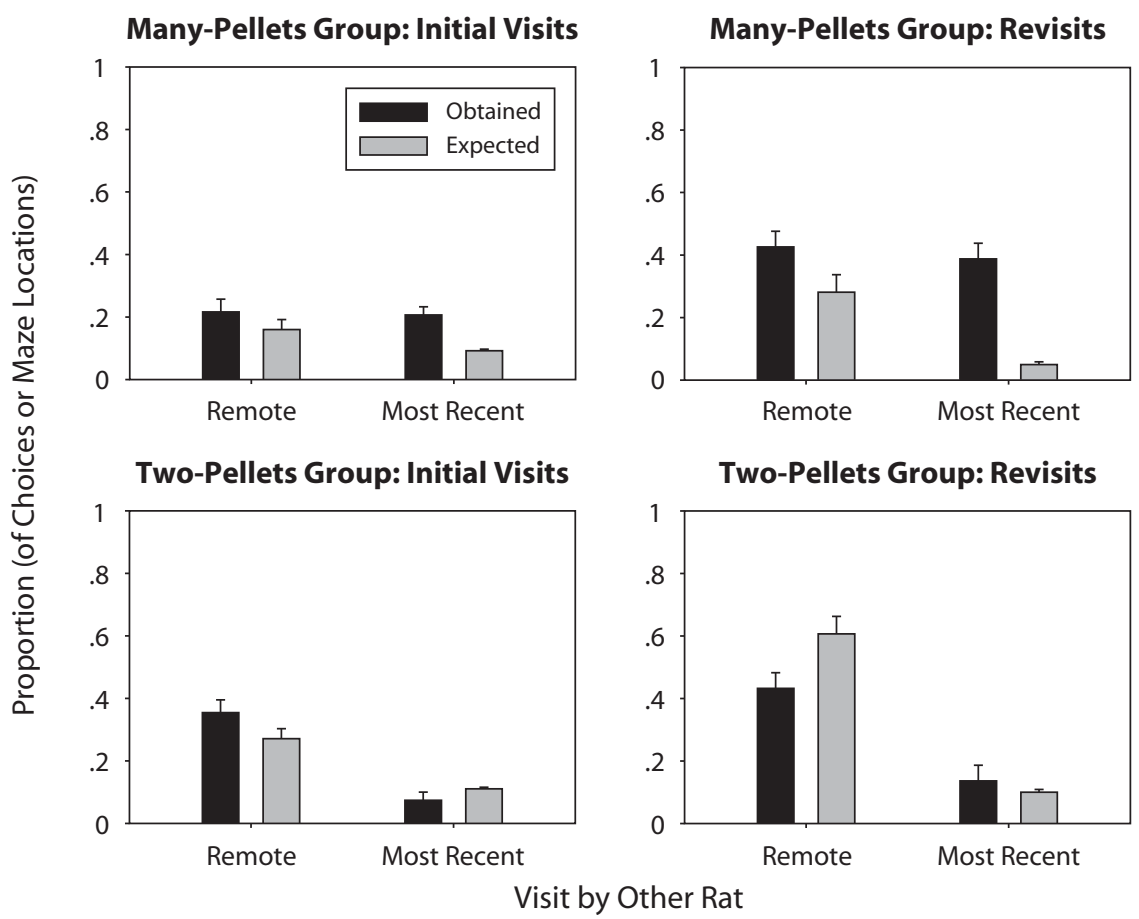

Figure 4. Mean proportions of maze locations chosen (obtained proportions) and proportions of maze locations available at the time those choices were made (expected proportions) in Experiment 4, for trials during which the maze-arm sleeves were absent (A) and present (B). Each panel shows the proportions for the most recent choice of the other rat and the choice made by the other rat earlier in the trial. The values shown are the means (over rats) of the resulting proportions for initial visits to a maze location by the focal rat (left panels) and revisits to maze locations by the focal rat (right panels) in the experimental group for which the maze locations were baited with many pellets (upper panels in both $A$ and B) or with two pellets (lower panels in both $A$ and B). Error bars show one standard error of the mean. 
Iable 2

Results of ANOVAs and $t$ ' l'ests for Experiment 4

\begin{tabular}{|c|c|c|c|c|c|c|}
\hline \multirow{2}{*}{$\begin{array}{l}\text { Food } \\
\text { Amount } \\
\text { Group }\end{array}$} & \multirow{2}{*}{$\begin{array}{c}\text { Arm } \\
\text { Sleeve } \\
\text { Condition }\end{array}$} & \multirow{2}{*}{$\begin{array}{c}\text { Focal Rat } \\
\text { Visit Status }\end{array}$} & \multirow[b]{2}{*}{ Proportion } & \multirow{2}{*}{$\begin{array}{c}\text { Other Rat Visit } \\
\text { Status } \times \\
\text { Proportion }\end{array}$} & \multicolumn{2}{|c|}{$t$ Test } \\
\hline & & & & & Remote & Recent \\
\hline Many pellets & No sleeves & Initiol vicita & $F(17)=56$ & $F(1,7)=3.4$ & & \\
\hline Many pellets & No sleeves & Revisits & $F(1,7)=13.8^{*}$ & $F(1,7)=12.4^{*}$ & $t(7)<1.0$ & $t(7)=5.7^{*}$ \\
\hline Two pellets & No sleeves & Initial visits & $F(1,7)=7.8^{*}$ & $F(1,7)=17.5^{*}$ & $t(7)=4.8^{*}$ & $t(7)=1.0$ \\
\hline Two pellets & No sleeves & Revisits & $F(1,7)<1.0$ & $F(1,7)=1.3$ & & \\
\hline Many pellets & Sleeves & Initial visits & $F(1,7)=55.8^{*}$ & $F(1,7)=1.9$ & & \\
\hline Many pellets & Sleeves & Revisits & $F(1,7)=34.3^{*}$ & $F(1,7)=3.3$ & & \\
\hline Two pellets & Sleeves & Initial visits & $F(1,7)=4.8$ & $F(1,7)=15.2^{*}$ & $t(7)=4.2^{*}$ & $t(7)=2.1$ \\
\hline Two pellets & Sleeves & Revisits & $F(1,7)=8.1^{*}$ & $F(1,7)=13.5^{*}$ & $t(7)=4.3^{*}$ & $t(7)=1.0$ \\
\hline
\end{tabular}

Note-Remote, remote choices of other rat; Recent, most recent choice of other rat. ${ }^{*} p<.05$.

As in Experiments 2 and 3, the overall proportion of choices of locations previously visited by the other rat (.277) was greater than the proportion expected on the basis of location available that had been visited by the other rat (.206) [proportion type, $F(1,14)=29.7$ ]. The significant interactions involving proportion type were proportion type $\times$ food amount $[F(1,14)=34.8]$, proportion $\times$ food amount $\times$ sleeves $[F(1,14)=5.3]$, proportion $\times$ food amount $\times$ focal status $[F(1,14)=20.4]$, proportion $\times$ other status $[F(1,14)=12.6]$, proportion $\times$ focal status $\times$ other status $[F(1,14)=21.15]$, and proportion $\times$ food amount $\times$ other status $[F(1,14)=6.8]$. In addition, the proportion $\times$ food amount $\times$ focal status $X$ sleeves interaction term was marginally significant $[F(1,14)=4.17, p=.06]$.

To understand the nature of these interactions, we conducted eight proportion type $\times$ other status ANOVAs, one for each level of sleeves and each level of focal status for each of the two food amount groups (i.e., one ANOVA corresponds to each of the panels shown in Figure 4). Significant effects of proportion or proportion $\times$ other status interactions are indicated in Table 2. There was a significant tendency to visit maze locations that had been visited by the other rat (indicated by a main effect of proportion) in six of the eight conditions and a significant interaction between other status and proportion in four of them. To determine the form of the interactions, $t$ tests were conducted comparing proportions of choices to maze locations visited by the other rat with the proportion expected. The results of these comparisons are shown in Table 2. A key finding is that rats in the two-pellets group chose maze locations that they had previously visited (i.e., revisits) and that were previously visited by the other rat less than would be expected by chance when the sleeves were present.

\section{Discussion}

The presence of the sleeves on the maze arms resulted in avoidance of maze locations that had been visited earlier in the trial by the other rat, but only in the two-pellets condition and only for maze locations that had been visited earlier by the focal rat. The fact that avoidance of locations visited earlier by the other rat was restricted to the two-pellets condition can be interpreted in terms of the contingency between a visit by the other rat and the presence of food in the maze location: In the two-pellets condition, food was no longer present in the maze location if the other rat had visited the location. Thus, this pattern of results is quite consistent with the ideas motivating the present experiments. When a visit by the other rat results in depletion of the food in a maze location, remembered visits by the other rat inhibit choice of that location.

The fact that this pattern of results occurred only for locations that had already been visited by the focal rat cannot be explained as readily. On one hand, revisits to maze arms by the focal rat are rare, and one would expect locations visited earlier by the focal rat to be avoided. On the other hand, only by visiting a maze arm is the focal rat exposed to information about whether that location is baited with two or with many pellets. That information may be critical for modulating the effect of the other rat's visits on subsequent choices of the focal rat. As detailed below, this interaction among the effects of focal rat visit status, food amount, and presence of sleeves suggests that information obtained by the focal rat during its initial visit to a location is combined with the social memory for the other rat's choice of that location to determine whether the other rat's choice increases or decreases that tendency of the focal rat to revisit that maze arm.

\section{GENERAL DISCUSSION}

The present experiments replicate the previous demonstrations from our laboratory of social influence on spatial choices in the radial-arm maze. In the present experiments, as well as in the earlier experiments reported by Brown et al. (2008), there was a consistent tendency to visit the location that was most recently visited by a foraging partner. In the cases of the location most recently visited by the other rat, the other rat is often still present in or near the location. Thus, we infer, the physical presence of another rat is an attractant stimulus that increases choices of the location occupied by (or near) the foraging partner.

Social influences of choices made earlier during the trial by the other rat must be explained by processes that persist over time. Although we did not control for the possibility that odor cues were involved in the present experiments, this explanation for social influences in the radial-arm maze was tested and ruled out by the previous experiments of Brown et al. (2007; see also Brown et al., 2008, pp. 336-337), and so we believe that the social influences on spatial choice in the radial-arm maze that per- 
sist over time are best explained in terms of memory for the earlier choices of the foraging partner. In the present experiments and in the earlier experiments from our laboratory, an effect of choices made earlier in the trial by the other rat was consistently present. However, the nature of that influence varied, sometimes producing an increased tendency to visit locations earlier visited by the foraging partner and sometimes producing the opposite tendency to avoid visits to those locations.

The former tendency (to visit locations that were visited by a foraging partner earlier during a trial) could be explained without any social effect of one rat on the other rat. If the rats have preferences for locations (maze arms) and those preferences are similar for the cagemate pairs tested together in these experiments, a tendency to visit the same locations would be produced. Similarity of location preferences, of course, could also be produced by social effects. If one or both rats in each pair have consistent location preferences and the rat with the preference influences the choices of the other rat, similar apparent preferences for maze location in the two rats would result. Clear evidence against the common location preference explanation of the tendency to visit locations that were previously visited by another rat is provided by the results of Experiment 1. A correlation between the orders in which maze locations are visited occurred only when the rats were tested together. The same rats tested in separate trials did not show a similarity of maze location preferences. This result rules out an explanation of the tendency to visit locations that were earlier visited by another rat in terms of common location preferences and demonstrates that the choices made by one rat change the tendencies of their foraging partner to visit locations.

In the present experiments, the effect of previous choices by the other rats was consistently an increased tendency to visit those locations, with one exception. In Experiment 4, with the maze arms rendered opaque by sleeves, arms visited earlier by the other rat were chosen less often than expected, but only if the focal rat had an opportunity to determine that they were depleted of food (i.e., they were baited with only two pellets and the focal rat had visited the maze arm earlier during the trial).

A possible interpretation of this pattern of results is as follows: If a foraging partner is visible in the maze, locations previously (as well as currently) visited by it are more likely to be visited. Assuming that the transparent maze arms render the foraging partner generally visible during trials, this can account for the increased tendency to visit locations visited by the other rat in Experiments 2 and 3, as well as in the no-sleeves conditions of Experiment 4 . In the sleeves conditions of Experiment 4, the other rat was present in or near its most recent choice, and so there was an increased tendency to visit those locations. In the case of the other rat's remote choices, however, the influence that they have on the focal rat's choices depends on the contingency between previous visits to the location and the presence of food. Locations with many food pellets were not depleted by previous visits, and so the increase in tendency to visit locations previously visited by the other rat remained. Locations with two pellets, on the other hand, remained baited only if neither rat had visited them previously during the trial. In correspondence with this contingency, locations with two pellets that were visited by the other rat and also visited by the focal rat were avoided. Otherwise, locations visited previously by the other rat were visited more often than expected.

This interpretation requires that information obtained during the rat's own visit to a maze location be combined with information about the behavior of its foraging partner. Brown et al. (2008) also found a pattern of results that required a combination of information from a rat's own visit and social information. In their experiments, the visits of another rat generally increased the tendency to visit maze arms, but the one exception was locations previously visited by the focal rat containing a less-preferred food type: Visits to those locations by the other rat decreased the tendency of the focal rat to visit them. Thus, a consistent finding is that the relatively low value of a maze location, as determined by the focal rat's visit to that location, combined with a remembered visit to that location by the other rat, decreases the tendency to choose that location.

There are parallels between these social effects on spatial working memory and long-term social influences on food preferences. Rats with little or no information about the value of two food types are influenced by the food choices made by conspecifics (e.g., Galef \& Giraldeau, 2001; Galef \& Wigmore, 1983). Similarly, the short-term, working-memory-based social influence in the present paradigm led to a general tendency to make choices that matched those of the foraging partner. Integration of information acquired socially with information about the same referent acquired personally has also been found in the context of long-term acquisition of food preferences by rats (Galef \& Whiskin, 1999), as well as guppies (Kendal, Coolen, \& Laland, 2004).

The social influence on choices in the radial-arm maze reported by Brown et al. (2007) was primarily a tendency to avoid visits to locations visited by the other rat. What accounts for the difference between that result and the opposite effect found in most conditions of the present experiments and those reported by Brown et al. (2008)? A definitive answer will require additional work, but a likely possibility is that the physical presence of the foraging partner modulates the influence of remembered choices made by it. In the forced choice procedure used by Brown et al. (2007), the foraging partner was removed from the apparatus before the focal rat made its choices. In those experiments, a tendency to avoid locations previously visited by the other rat was found. When the other rat was present as the focal rat made its choices, the focal rat had a tendency to visit the location of its most recent choice (where it was likely to still be present) but a tendency to avoid choices it made earlier during the trial. Critically, these experiments involved a maze constructed of opaque material, so the other rat was generally not visible except in or near its most recent choice. In the more recent experiments using a maze constructed of transparent material, previous visits by the other rat increased the tendency to visit maze locations. Assuming that the transparent ma- 
terial increases the extent to which the presence of the other rat is generally detected, this is consistent with the idea that social presence modulates the effect of social memories. Memories of visits by another rat decrease the tendency to visit those locations if the rat is not currently present but increase the tendency to visit those locations if the rat is currently present.

It is somewhat surprising that the dominant effect of previous visits by another rat is to increase the relative likelihood of visiting the maze location, given the ubiquitous finding that the rats avoid revisits to maze locations that they have, themselves, visited and that the effect occurs despite a contingency between visits by the other rat and depletion of food at that location. It appears that a strong social facilitation effect is produced by the presence of another rat (at least in the case of a familiar cagemate). As long as there are sufficient cues indicating the presence of the other rat, memory for its spatial choices produces an increased tendency to visit those locations. Only when those social cues are weakened does the more familiar avoidance of previously chosen locations occur, specifically in the observer/model procedures used by Brown et al. (2007), in which the other rat was removed from the maze before the focal rat made its choices, and in the conditions involving the opaque maze arms that reduced visual cues regarding the other rat's presence (Experiment 4). Rats remember choices made both by themselves and by a foraging partner, but when there are sufficient cues that the foraging partner is present, those memories have opposite effects on behavior.

The results of these experiments provide direct support for three conclusions and suggest one additional possibility. First, increased tendencies to visit locations previously visited by another rat- just like decreased tendencies to do so-result from a social influence of one rat's choices on those made by its foraging partner. Second, we find no evidence that the amount of food available in a maze location, by itself, determines the behavioral outcome of social working memory. Generally, rats tend to visit locations previously visited by another rat regardless of the contingency between its visit and the subsequent availability of food there. Third, social effects on spatial choice interact with working memory produced by the rat's own visits, such that places visited earlier by another rat and also visited by the focal rat itself influence its subsequent choices differently, depending on the amount of food available there. Finally, the present results considered together with earlier results from our laboratory suggest a more general process by which information about spatial locations obtained by a rat's own visits is combined with social memories for the visits of a foraging partner to determine spatial choice.

\section{AUTHOR NOTE}

Experiment 1 was the basis of an M.S. thesis submitted to Villanova University by T.-M.N.P., who is currently at the University of Pennsylvania. K.E.D. is currently at Kent State University. Correspondence should be addressed to M. F. Brown, Department of Psychology, Villanova University, Villanova, PA 19085 (e-mail: michael.brown@ villanova.edu).

\section{REFERENCES}

Brown, M. F., Farley, R. F., \& Lorek, E. J. (2007). Remembrance of places you passed: Social spatial working memory in rats. Journal of Experimental Psychology: Animal Behavior Processes, 33, 213-224. doi:10.1037/0097-7403.33.3.213

Brown, M. F., Knight-Green, M. B., Lorek, E. J., Packard, C., Shallcross, W., Wifall, T., ET AL. (2008). Social working memory: Memory for another rat's choices can increase or decrease choice tendencies. Learning \& Behavior, 36, 327-340. doi:10.3758/ LB.36.4.327

Galef, B. G., JR., \& Giraldeau, L. A. (2001). Social influences on foraging in vertebrates: Causal mechanisms and adaptive functions. Animal Behaviour, 61, 3-15. doi:10.1006/anbe.2000.1557

GALEF, B. G., JR., \& LALAND, K. N. (2005). Social learning in animals: Empirical studies and theoretical models. BioScience, 55, 489-499.

GALEF, B. G., JR., \& Whiskin, E. E. (1999). Use of public information when foraging: Effects of time available to sample foods. Animal Cognition, 2, 103-107. doi:10.1007/s100710050030

Galef, B. G., JR., \& Wigmore, S. W. (1983). Transfer of information concerning distant foods: A laboratory investigation of the "information-centre" hypothesis. Animal Behaviour, 31, 748-758.

Kendal, R. L., Coolen, I., \& Laland, K. N. (2004). The role of conformity in foraging when personal and social information conflict. Behavioral Ecology, 15, 269-277. doi:10.1093/beheco/arh008

Olton, D. S. (1978). Characteristics of spatial memory. In S. H. Hulse, H. Fowler, \& W. K. Honig (Eds.), Cognitive processes in animal behavior (pp. 341-374). Hillsdale, NJ: Erlbaum.

Olton, D. S., \& Samuelson, R. J. (1976). Remembrance of places passed: Spatial memory in rats. Journal of Experimental Psychology: Animal Behavior Processes, 2, 97-116.

Zentall, T. R., \& Galef, B. G., Jr. (Eds.) (1988). Social learning: Psychological and biological perspectives. Hillsdale, NJ: Erlbaum.

(Manuscript received August 12, 2008; revision accepted for publication January 29, 2009.) 